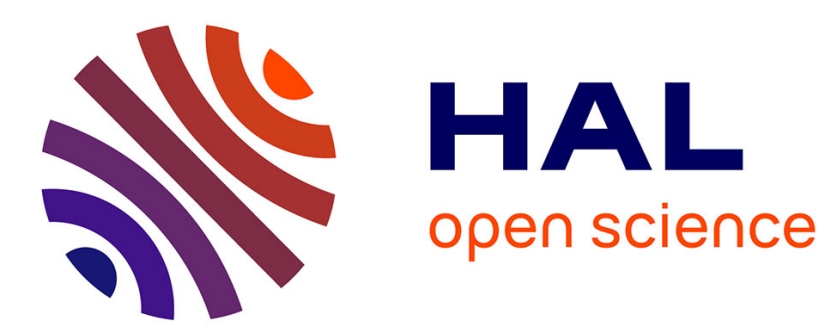

\title{
Periodic controls for discriminating density dependent growth in the chemostat
}

Fatima Zahra Tani, Alain Rapaport, Térence Bayen

\section{To cite this version:}

Fatima Zahra Tani, Alain Rapaport, Térence Bayen. Periodic controls for discriminating density dependent growth in the chemostat. IEEE Conference on Decision and Control CDC2019, IEEE, Dec 2019, Nice, France. pp.4735-4740, 10.1109/CDC40024.2019.9029891 . hal-02433700

\section{HAL Id: hal-02433700 https://hal.science/hal-02433700}

Submitted on 9 Jan 2020

HAL is a multi-disciplinary open access archive for the deposit and dissemination of scientific research documents, whether they are published or not. The documents may come from teaching and research institutions in France or abroad, or from public or private research centers.
L'archive ouverte pluridisciplinaire HAL, est destinée au dépôt et à la diffusion de documents scientifiques de niveau recherche, publiés ou non, émanant des établissements d'enseignement et de recherche français ou étrangers, des laboratoires publics ou privés. 


\title{
Periodic controls for discriminating density dependent growth in the chemostat
}

\author{
Fatima-Zahra Tani, Alain Rapaport and Térence Bayen
}

\begin{abstract}
We show the benefit of considering periodic dilution rates in the chemostat model for discriminating between a growth function which does not depend on the density of the micro-organisms population (such as the Monod law) and another one which does depend (such as the Contois law). This goal is achieved thanks to the measurement of the abiotic resource only. We then present a simple procedure for a robust discrimination between the two types of kinetics using a single experiment in three phases. Finally, the shape of the best periodic excitation is discussed and the method is illustrated on numerical simulations.
\end{abstract}

\section{INTRODUCTION}

Continuous culture has been invented simultaneously by Monod [23] and Novick \& Szilard [25] in the fifties as a means to measure accurately growth rates of microorganisms. Typically, the so-called chemostat device consists in feeding a culture vessel of volume $V$ with a nutritive solution of concentration $s_{i n}$ at a constant flow rate $Q$, and then in extracting micro-organisms and substrate at the same rate $Q$ from the culture vessel (so that the volume remains constant). The concentrations of micro-organisms and substrate, denoted respectively by $b$ and $s$ are then solutions of the following differential equations, that reflect the writing of the mass balance

$$
\begin{aligned}
\dot{s} & =-\frac{1}{Y} \mu(s) b+D\left(s_{i n}-s\right), \\
\dot{b} & =\mu(s) b-D b,
\end{aligned}
$$

where $D:=Q / V$ denotes the dilution rate, $Y$ the conversion rate and $\mu(\cdot)$ the specific growth rate. Each bacterial species is characterized by the positive constant $Y$ and the nonnegative function $s \mapsto \mu(s)$ satisfies $\mu(0)=0$. The usual way to identify $Y$ and $\mu(\cdot)$ are as follows.

1. The parameter $Y$ is obtained from the batch mode which consists in taking $D=0$ (see for instance [12]). One can check that solutions of (1) with positive initial conditions $\left(s_{0}, b_{0}\right)$ converge asymptotically to the steady state $\left(s^{\star}, b^{\star}\right):=$ $\left(0, b_{0}+Y b_{0}\right)$. The parameter $Y$ is thus determined by

$$
Y=\frac{b^{\star}-b_{0}}{s_{0}}, s
$$

which is usually considered as a robust method.

F.-Z. Tani and A. Rapaport are with MISTEA, Univ. Montpellier, INRA, Montpellier SupAgro, France alain.rapaporteinra.fr, fatima-zahra.taniesupagro.fr

T. Bayen is with Avignon Université, Laboratoire de Mathématiques d'Avignon (EA 2151) F-84018 Avignon, France terence.bayen@univ-avignon. fr
2. There are two general approaches for estimating the growth rate function $\mu(\cdot)$ : either by adjusting the dynamical model to growth curves (in batch or in continuous culture) [6], [14], either by a series of steady-states. The first method is often considered less accurate because it deals with transient dynamics and biomass sampling can perturb the culture. The second method measures steady-states only, for which cell density no longer change with time (historically this method owes its name to "chemostat"), and is considered more reliable [19]. The growth function $\mu(\cdot)$ is then identified step by step, considering a series of experiments with different constant values of $D$ (see for instance [29]). One can easily check that positive equilibria $\left(s^{\star}, b^{\star}\right)$ of (1) are given by $\mu\left(s^{\star}\right)=D$ and $b^{\star}=Y\left(s_{i n}-s^{\star}\right)$, when $s^{\star}$ is below $s_{i n}$. Therefore each value of $D$ provides a point $\left(s^{\star}, D\right)$ of the graph of $\mu(\cdot)$. This method implicitly assumes that there exists a unique solution $s$ to the equation $\mu(s)=D$ and that the corresponding steady state is asymptotically stable. One can easily show (see for instance [18]) that this is fulfilled when $\mu(\cdot)$ is monotone and $D$ is chosen less than $\mu\left(s_{i n}\right)$. For non-monotonic growth functions, other methods can be used (see for instance [27]).

Most often, the growth functions that suit data are concave increasing, such as the Monod law [23]:

$$
\mu_{M}(s):=\mu_{\max } \frac{s}{K_{s}+s} .
$$

However, the function $\mu$, differently to the parameter $Y$, might depend on environmental growth conditions (temperature, $\mathrm{pH}, \ldots$.). Parameters $\mu_{\max }, K_{s}$ could then depend on experiments and measurements. It also happens that the growth is inhibited by large concentrations $b$ of the biomass, reflecting a crowding effect of micro-organisms. This is particularly met in bioreactors of industrial wastewater plants. Then, one has to look for density dependent functions, i.e., of the form $(s, b) \mapsto \mu(s, b)$, increasing with respect to $s$ and non-increasing with respect to $b$. The most popular one is the Contois law [11], given by the following expression

$$
\mu_{C}(s, b):=\mu_{\max } \frac{s}{s+k b} .
$$

Notice that Contois law can be interpreted as a Monod expression but applied to the ratio $s / b$ instead of $s$. However it does not generalize the Monod function for small density effect, but one can consider the generalized Contois law

$$
\mu_{C G}(s, b):=\mu_{\max } \frac{s}{K_{s}+s+k b},
$$


to recover Monod law (taking $k=0$ ) and Contois law (taking $K_{s}=0$ ) expressions. Here the parameter $k>0$ measures the density effect. Recently, another expression of density dependent function has been proposed and experimentally validated [20]:

$$
\mu_{K}(s, b):=\mu_{0} \frac{s}{b^{\alpha}}
$$

where $\alpha$ is a non-negative parameter.

Before setting up a model identification and control strategies, it is worth to mention that the choice between Monod law or density dependent extensions has important impacts on the predictions of the behavior of bacterial species (the greater the parameter $k$ or $\alpha$ is, the greater the impacts are). First of all, steady state concentrations of substrate do not depend on the input concentration $s_{\text {in }}$ in the case of Monod law, while it does in the case of Contois law [18]. This is clearly an issue for the piloting of waste-water plants for which the input concentration $s_{\text {in }}$ fluctuates with time. Secondly, the Competitive Exclusion Principle [28], [18] applies when the growth functions depend on $s$ only: it asserts that only one species coexists in the long run in the chemostat model. On the contrary, a dependency on biomass concentration in the kinetics can allow coexistence of several species [21], [10], [22], which can impact the performances of bioreactors. Therefore, the discrimination between substrate and ratio dependency is a question of prime importance, in particular for the choice of sensors and control laws.

The classical identification method we recalled previously imposes many experiments to reconstruct accurately the graph of $\mu$ (as one experiment provides only one point of its graph) and can be then lengthy and costly, whereas control laws do not necessarily require a perfect knowledge of the function $\mu$ (when it depends on $s$ only, see for instance [6], [26], [3]). Notice that the identification of density dependent functions requires the additional measurement of the biomass, which is often more difficult and expensive to obtain than the abiotic component. Ratio-dependency has received a great attention in the ecological literature (see for instance [4]), and a conceptual experiment of several tanks in series has been designed to qualitatively discriminate ratiodependency in the context of prey-predators [5]. Inspired by this approach, a similar experiment has been investigated in the context of bioreactors [17]. However, it imposes to be able to retain perfectly the biomass from one reactor to the following one, which can be a practical issue.

The purpose of the present work is to investigate a robust methodology for discriminating between the two kinds of growth functions, on the basis of a simple experiment with a single tank and the single measurement of the substrate. Instead of steady state operations, as in the methods described above, we propose to control the experiment with periodic dilution rate $D(\cdot)$ instead of constant ones. Periodic operations of chemostat have already been investigated in the literature, but for optimizing performances when the growth function is known (see for instance [1], [2]).
The paper is organized as follows. In Sec. II, we give results about periodic solutions of the chemostat model with periodic controls. In particular, we show in Proposition 2 the role played by the convexity of $\mu(\cdot)$ in the average value of $s(\cdot)$ over a period, compared to a steady state $s^{\star}$. This result is the core of the procedure that is presented in Sec. III. In this section, we first define "weak" and "strong" density dependent effects, and we then give an experiment procedure based on bang-bang controls for discriminating between these two aforementioned cases with the single measurement of the variable $s$ only. In Sec. IV, we justify the choice of bangbang controls as the optimal ones. Finally, Sec. V illustrates this methodology on numerical simulations showing also the robustness of the method when environmental conditions could suddenly change.

\section{THE CHEMOSTAT MODEL UNDER PERIODIC CONTROL}

In the sequel, we shall consider non-negative measurable control functions $D: \mathbb{R}_{+} \rightarrow \mathbb{R}_{+}$such that:

- the control $D(\cdot)$ is periodic of period $T$ (to be chosen), - the control $D(\cdot)$ is persistently exciting ([6]) and satisfies

$$
\frac{1}{T} \int_{0}^{T} D(t) d t=\bar{D}>0,
$$

where $\bar{D}>0$ is given. Considering the total mass concentration $m=Y s+b$, (1) implies that

$$
\dot{m}=D(t)\left(Y s_{\text {in }}-m\right) .
$$

Then, any periodic solution of (1) has to fulfill $m(t)=Y s_{\text {in }}$ for any time $t$. Therefore, we shall consider the reduced dynamics on the invariant line $Y s+b=Y s_{i n}$, that is, the scalar dynamics

$$
\dot{s}=F(s, D):=\left(s_{\text {in }}-s\right)(D-v(s)),
$$

on the invariant interval $I:=\left(0, s_{i n}\right)$, where the function $v$ is defined as follows

$$
v(s):= \begin{cases}\mu(s) & \text { if } \mu=\mu_{M}, \\ \mu\left(s, Y\left(s_{\text {in }}-s\right)\right) & \text { if } \mu=\mu_{C G} .\end{cases}
$$

Notice that in any case, the function $v$ is increasing. Therefore, for $\bar{D}<v\left(s_{i n}\right)$, there exists a unique solution $s^{\star}$ of

$$
v\left(s^{\star}\right)=\bar{D},
$$

with $x^{\star}:=Y\left(s_{\text {in }}-s^{\star}\right)>0$, and $\left(s^{\star}, x^{\star}\right)$ is thus the unique asymptotically stable equilibrium of (1) for the constant control $D=\bar{D}$. Let us define for convenience the function

$$
\lambda(D):= \begin{cases}v^{-1}(D) & \text { if } D<v\left(s_{i n}\right), \\ s_{\text {in }} & \text { otherwise. }\end{cases}
$$

We start by giving a result about the convergence of solutions of (7) to a unique $T$-periodic solution, using the usual argumentation for scalar dynamics based on the Poincaré mapping (see for instance [28]).

Lemma 1: Suppose that $\bar{D}<v\left(s_{\text {in }}\right)$. Then, for any $T$ periodic control $D(\cdot)$ that satisfies (6) and any initial condition $s_{0} \in I$, the associated solution of (7) converges asymptotically to the unique $T$-periodic solution of (7) associated with $D(\cdot)$. 
Proof: For a given $T$-periodic control $D(\cdot)$, consider the $C^{1}$ function $\phi: I \mapsto I$ defined by $\phi\left(s_{0}\right):=s\left(T, s_{0}\right)$ where $s\left(\cdot, s_{0}\right)$ denotes the solution of (7) for the initial condition $s_{0}$ at time 0 . By differentiating $\phi$, one has

$$
\phi^{\prime}\left(s_{0}\right)=\frac{\partial s}{\partial s_{0}}\left(T, s_{0}\right)=X(T)
$$

where $X(\cdot)$ is the fundamental solution of the variational equation $\dot{z}=\frac{\partial f}{\partial s}\left(s\left(t, s_{0}\right), D(t)\right) z$ with $z(0)=1$. One gets:

$$
\phi^{\prime}\left(s_{0}\right)=e^{-\int_{0}^{T}\left[D(t)-v\left(s\left(t, s_{0}\right)\right)+v^{\prime}\left(s\left(t, s_{0}\right)\right)\left(s_{i n}-s\left(t, s_{0}\right)\right)\right] d t} .
$$

Clearly, $\phi^{\prime}>0$ and thus $\phi$ is increasing. For $s_{0}=0$, the solution cannot be identically equal to 0 because of condition (6) and $F(0, D) \geq 0$. Therefore one has $\phi(0)=s(0, T)>0$. For $s_{0}=s_{i n}$, the solution of (7) is constant equal to $s_{i n}$. Then $\phi\left(s_{\text {in }}\right)=s_{\text {in }}$ and

$$
\phi^{\prime}\left(s_{\text {in }}\right)=e^{-\int_{0}^{T}\left[D(t)-v\left(s_{i n}\right)\right] d t} .
$$

Since $\bar{D}<v\left(s_{\text {in }}\right)$, we deduce that $\phi^{\prime}\left(s_{\text {in }}\right)>1$. Consequently, the $C^{1}$ function $s_{0} \mapsto \psi\left(s_{0}\right):=\phi\left(s_{0}\right)-s_{0}$ satisfies $\psi(0)>0$, $\psi\left(s_{i n}\right)=0$, and $\psi^{\prime}\left(s_{i n}\right)>0$, thus it has necessarily a zero in $I$ which proves the existence of a fixed point $\bar{s}_{0}$ of $\phi$ as well as the existence of a $T$-periodic solution $\bar{s}(\cdot):=s\left(\cdot, \bar{s}_{0}\right)$ of (7). Moreover, at $s_{0}=\bar{s}_{0}$, one finds

$$
\psi^{\prime}\left(\bar{s}_{0}\right)=e^{-\int_{0}^{T}\left[D(t)-v(\bar{s}(t))+v^{\prime}(\bar{s}(t))\left(s_{\text {in }}-\bar{s}(t)\right] d t\right.}-1 .
$$

Observe now that for a periodic solution $\bar{s}(\cdot)$, one has

$$
0=\ln \left(\frac{s_{\text {in }}-\bar{s}(T)}{s_{\text {in }}-\bar{s}_{0}}\right)=-\int_{0}^{T}[D(t)-v(\bar{s}(t))] d t
$$

from which one obtains

$$
\psi^{\prime}\left(\bar{s}_{0}\right)=e^{-\int_{0}^{T} v^{\prime}(\bar{s}(t))\left(s_{i n}-\bar{s}(t)\right) d t}-1<0 .
$$

Since at every point $\bar{s}_{0} \in I$ such that $\psi\left(s_{0}\right)=0$, one has $\psi^{\prime}\left(\bar{s}_{0}\right)<0$, we then conclude that $\psi$ has exactly one zero $\bar{s}_{0} \in I$ which shows the uniqueness of the $T$-periodic solution $\bar{s}(\cdot)$.

Notice that $\bar{s}(\cdot)$ is asymptotically stable since $\phi^{\prime}\left(\bar{s}_{0}\right)<$ 1 (see [28]). Finally, for any $s_{0} \in I \backslash\left\{\bar{s}_{0}\right\}$, the sequence $\left(\phi^{n}\left(s_{0}\right)\right)_{n}$ is monotone since $\phi$ is increasing, and thus it converges to a fixed point of $\phi$. The only fixed point at boundary of $I$ is $s_{\text {in }}$ with $\phi^{\prime}\left(s_{i n}\right)<1$ which implies that $\phi\left(s_{0}\right)<s_{0}$ for $s_{0}$ close to $s_{i n}$. One can verify that $\phi^{n}\left(s_{0}\right)$ necessarily converges to $\bar{s}_{0}$ when $n$ goes to infinity which implies that $\left|s\left(t, s_{0}\right)-\bar{s}(t)\right| \rightarrow 0$ when $t$ goes to infinity.

The next result will play an important role in the following. The key point is to consider functions $D(\cdot)$ that fulfill (6) for the same value $\bar{D}$. Hereafter, for a given non constant $T$-periodic control $D(\cdot)$ that fulfills (6), we denote by $\bar{s}(\cdot)$ the unique periodic solution associated with $D(\cdot)$.

Proposition 2: If the restriction of $v(\cdot)$ to the interval $J:=$ $\{v(\bar{s}(t)), t \in[0, T]\}$ is convex, respectively concave, and does not coincide with a linear function on this interval, then

$\hat{s}:=\frac{1}{T} \int_{0}^{T} \bar{s}(t) d t<s^{\star}, \quad$ respectively $\hat{s}:=\frac{1}{T} \int_{0}^{T} \bar{s}(t) d t>s^{\star}$.
Proof: Let $\bar{s}(\cdot)$ be a non-constant $T$-periodic solution with values in $I$. From (8), we deduce the equality

$$
\frac{1}{T} \int_{0}^{T} v(\bar{s}(t)) d t=\bar{D}
$$

When $v$ is convex on the interval $J$, applying Jensen's inequality to $v$ yields

$$
v\left(\frac{1}{T} \int_{0}^{T} \bar{s}(t) d t\right) \leq \frac{1}{T} \int_{0}^{T} v(\bar{s}(t)) d t
$$

Similarly, when $v$ is concave on $J$, one obtains the reversed inequality. Since equality in Jensen's inequality holds true if and only if $v$ is affine over $J$, the inequality in (10) is strict. Combining (9)-(10) then gives

$$
v\left(\frac{1}{T} \int_{0}^{T} \bar{s}(t) d t\right)<v(\bar{s})
$$

and, as $v$ is increasing, we get

$$
\frac{1}{T} \int_{0}^{T} \bar{s}(t) d t<s^{\star}
$$

The reversed inequality is obtained in the same way when $v$ is concave over $J$.

Lemma 3: The solution $\bar{s}$ verifies the inequalities:

$$
\min _{t \in[0, T]} \bar{s}(t) \leq s^{\star} \leq \max _{t \in[0, T]} \bar{s}(t)
$$

Proof: Equality (9) can be equivalently written

$$
\frac{1}{T} \int_{0}^{T}\left[v(\bar{s}(t))-v\left(s^{\star}\right)\right] d t=0
$$

Then, the function $t \mapsto v(\bar{s}(t))-v\left(s^{\star}\right)$ has to change its sign over $[0, T]$. Since $v$ is monotone over $I$, we deduce that there exists $t_{-}$and $t_{+}$in $[0, T]$ such that $\bar{s}\left(t_{-}\right)<s^{\star}<\bar{s}\left(t_{+}\right)$.

Without any loss of generality, we can then consider periodic solutions of (7) with periodic controls satisfying (6) and such that $\bar{s}(0)=s^{\star}$. The constant solution $s=s^{\star}$ for constant $D=\bar{D}$ is one of them. We show how to use Prop. 2 to discriminate density-dependency.

\section{A DISCRIMINATING PROCEDURE}

Consider the growth functions $v$ associated to Monod and (generalized) Contois laws (following the notation of Sec. II):

$$
v_{M}(s):=\mu_{\max } \frac{s}{K_{s}+s} ; v_{C G}(s):=\mu_{\max } \frac{s}{K_{s}+s+k Y\left(s_{\text {in }}-s\right)} .
$$

One can easily check that these functions are increasing. A straightforward computation also gives

$$
v_{M}^{\prime \prime}(s)=\frac{-2 K_{s} \mu_{\max }}{\left(K_{s}+s\right)^{3}} ; v_{C G}^{\prime \prime}(s)=\mu_{\max } \frac{2(k Y-1)\left(K_{s}+k Y s_{\text {in }}\right)}{\left(K_{s}+s+k Y\left(s_{\text {in }}-s\right)\right)^{3}} \text {. }
$$

One can observe that $v_{M}$ is always concave, while $v_{C G}$ is convex when $k Y>1$. We shall say that the growth rate $v$ has a weak (or null) density effect if it follows the laws of Monod or (generalized) Contois with $k \leq 1 / Y$. Conversely, we shall say that it has a strong density effect if it follows the law of (generalized) Contois with $k>1 / Y$.

We investigate now how to use the results of Proposition 2 to discriminate between convex and concave functions using 
periodic controls. Let us underline that for a given value of $s^{\star}$, determining periodic non constant functions $D(\cdot)$ such that the solution $s(\cdot)$ with $s(0)=s^{\star}$ is periodic requires the perfect knowledge of the function $\mu$ and the constants $Y$ and $s_{i n}$. On another hand, waiting for the convergence to a periodic solution could be long and difficult to test in practice. We propose below an experiment procedure to discriminate between weak and strong density effects.

Procedure : It consists in conducting a single chemostat experiment with three phases: constant, bang-bang and constant. It requires the single measurement of the substrate $s$.

- Phase 1. Conduct an experiment for a constant dilution rate $D$ with $0<D<v\left(s_{i n}\right)$ (to avoid the washout equilibrium) and wait the chemostat to be at (quasi) steady state. Let $s^{e q}$ be the measurement of $s$, and $t_{0}$ the current time.

- Phase 2. Choose two values $D_{\min }, D_{\max }$ such that $0 \leq$ $D_{\min }<D<D_{\max }$, and two time durations $\delta_{1}>0, \delta_{2}>0$. Apply first $D=D_{\max }$ on the time interval $\left[t_{0}, t_{0}+\delta_{1}\right)$. Next, apply $D=D_{\text {min }}$ until the time $\bar{t}$ such that $s(\bar{t})=s_{e q}$, and carry on applying $D=D_{\min }$ during the duration $\delta_{2}$. Finally, change the flow rate value to $D=D_{\max }$ for $t>\bar{t}+\delta_{2}$. Store the measurements history $\{s(t)\}_{t \in\left[t_{0}, t_{0}+T\right]}$ until the first time $t_{0}+T>\bar{t}+\delta_{2}$ such that $s\left(t_{0}+T\right)=s^{e q}$ (which exists as $0 \leq D_{\min }<D<D_{\max }$ ). To summarize, one applies

$$
D(t):= \begin{cases}D_{\max }, & t \in\left[t_{0}, t_{0}+\delta_{1}\right), \\ D_{\min }, & t \in\left[t_{0}+\delta_{1}, \bar{t}+\delta_{2}\right), \\ D_{\max }, & t \in\left[\bar{t}+\delta_{2}, t_{0}+T\right] .\end{cases}
$$

Let $\bar{D}$ be the mean value of the dilution rate over $\left[t_{0}, t_{0}+T\right]$ :

$$
\bar{D}:=\frac{D_{\max }\left(T+t_{0}-\bar{t}+\delta_{1}-\delta_{2}\right)+D_{\min }\left(\bar{t}-t_{0}+\delta_{2}-\delta_{1}\right)}{T} .
$$

- Phase 3. Apply for $t>t_{0}+T$ the constant dilution rate $D:=\bar{D}$ and wait for the (quasi) steady state, denoted by $s^{\star}$. With the data stored on $\left[t_{0}, t_{0}+T\right]$, determine the average value of $s$ on $\left[t_{0}, t_{0}+T\right]$ :

$$
\hat{s}:=\frac{1}{T} \int_{t_{0}}^{t_{0}+T} s(t) d t .
$$

Conclusion. If $\hat{s}<s^{\star}$, we validate the strong density effect.

Phase 1 allows the chemostat to be at steady state, so that dynamics (7) is considered to be valid at time $t_{0}$. The phase 2 generates a $T$-periodic solution with a periodic control, over a single period, without having to wait the convergence to the periodic solution. Phase 3 compares the average value $\hat{s}$ of the substrate concentration on the periodic solution with the steady state one $s^{\star}$ obtained for a constant control $\bar{D}$ equal to the average value of the periodic control. Proposition (2) allows to conclude.

Remark 1: Note that $s^{\star}$ has no a priori reason to be equal to $s^{e q}$, as the initial value of $D$ is arbitrary.

Remark 2: This procedure is a way to discriminate between Monod and (generalized) Contois laws. It allows also to discriminate between convex or concave functions on the invariant interval $\left(\lambda\left(D_{\min }\right), \lambda\left(D_{\max }\right)\right)$ and can then be applied to other situations whose growth is expected to follow expressions such as (5). For this function, one has

$$
v_{K}^{\prime \prime}(s)=\alpha \mu_{0} \frac{2 s_{i n}+(\alpha-1) s}{\left(s_{i n}-s\right)^{\alpha+2}} .
$$

Therefore, $v_{K}$ is convex when $\alpha$ is larger than one (which has been observed in [20]) or when $s_{i n}$ is large enough.

The proposed procedure considers bang-bang periodic controls for discriminating convex functions $v$. One may wonder if the periodic control that satisfies (6) allows to obtain the largest difference between $\hat{s}$ and $s^{\star}$. We justify this choice in the next section.

\section{THE BEST SHAPE OF PERIODIC CONTROLS}

The goal of this section is to justify the construction of $D$ in (11) as a bang-bang control, in terms of optimal control. Given bounds $D_{\min }, D_{\max }$ on the control $D(\cdot)$ with $0 \leq D_{\min }<$ $\bar{D}<D_{\max }$, and given a convex function $v$, we look for $T$ periodic controls $D(\cdot)$ that satisfy (6) with

$$
D(t) \in\left[D_{\min }, D_{\max }\right] \quad \text { a.e. } t \geq 0,
$$

such that the associated solution $\bar{s}$ with $\bar{s}(0)=s^{\star}$ is $T$ periodic and gives the smallest average value $\hat{s}$ :

$$
\min _{D(\cdot)} \hat{s}:=\frac{1}{T} \int_{0}^{T} s(t) d t
$$

Similarly, when $v$ is concave, we would look for controls $D(\cdot)$ such that $\hat{s}$ is the largest. Problem (13) is an optimal periodic control problem. Several contributions about the theory of optimal control with periodic controls are available in the literature (see for instance [9], [15], [16] and references herein). However, in the present case, it has to fulfill the additional integral constraint (6), which has not been yet studied, up to our knowledge, expect in the recent works [7], [8].

Let $K:=\left(\lambda\left(D_{\min }\right), \lambda\left(D_{\max }\right)\right)$ and introduce the function $\eta: K \rightarrow \mathbb{R}_{+}^{*}$ as

$$
\eta(s):=\frac{1}{\left(s_{\text {in }}-s\right)\left(D_{\max }-v(s)\right)\left(v(s)-D_{\min }\right)}, s \in K .
$$

One has the following optimality result.

Proposition 4: Assume $v$ to be convex increasing on $K$, and take arbitrarily $\bar{D} \in\left(D_{\min }, D_{\max }\right)$ and $T>0$. Then:

(i) There are exactly two $T$-periodic optimal solutions of (13) under the constraints (6) and (12), and such that $s(0)=s^{\star}$.

(ii) The controls $D^{ \pm}$generate the two optimal trajectories (with same cost):

$$
\begin{gathered}
D^{+}(t):=\left\{\begin{array}{l}
D_{\text {max }}, t \in\left[0, t_{1}^{+}\right) \cup\left[t_{2}^{+}, T\right) \bmod T, \\
D_{\text {min }}, t \in\left[t_{1}^{+}, t_{2}^{+}\right) \bmod T,
\end{array}\right. \\
D^{-}(t):=\left\{\begin{array}{l}
D_{\text {min }}, t \in\left[0, t_{1}^{-}\right) \cup\left[t_{2}^{-}, T\right) \bmod T, \\
D_{\text {max }}, t \in\left[t_{1}^{-}, t_{2}-\right) \bmod T,
\end{array}\right.
\end{gathered}
$$

where times $t_{i}^{ \pm}, i=1,2$ are given by:

$$
\begin{aligned}
& t_{1}^{+}:=\inf \left\{t \geq 0 ; s(t)=s_{M}\right\}, t_{2}^{+}:=\inf \left\{t \geq t_{1}^{+} ; s(t)=s_{m}\right\}, \\
& t_{1}^{-}:=\inf \left\{t \geq 0 ; s(t)=s_{m}\right\}, t_{2}^{-}:=\inf \left\{t \geq t_{1}^{-} ; s(t)=s_{M}\right\},
\end{aligned}
$$


and where $\left(s_{m}, s_{M}\right)$ is the unique pair in $K^{2}$ that satisfies

$$
\int_{s_{m}}^{s_{M}} \eta(s) d s=\frac{T}{D_{\max }-D_{\min }}, \int_{s_{m}}^{s_{M}} \eta(s) v(s) d s=\frac{\bar{D} T}{D_{\max }-D_{\min }} .
$$

Proof: We apply a result of [7] that we recall in the Appendix. One can easily check that (13) is a particular instance of (18) taking $I^{\prime}=K, \tilde{\eta}=\eta, f(s)=\left(s_{\text {in }}-\right.$ $s)\left[\frac{D_{\max }+D_{\min }}{2}-v(s)\right], g(s)=\left(s_{i n}-s\right) \frac{D_{\max }-D_{\min }}{2}$ and $\ell(s)=s$, and verify that the required assumptions to apply the result of the Appendix are satisfied. The optimal solutions $u^{ \pm}$of (18) provided by (20) once translated into (13), lead to the optimal solutions $D^{ \pm}$of (13) given by (14)-(15). As well, (16) straightforwardly follows from (19).

This result gives the existence of $\delta_{1}, \delta_{2}$ that give the largest value $\left|\hat{s}-s^{\star}\right|$ in the procedure of Sec. III. Let us underline that $s^{\star}$ is not known in advance (as the function $v$ is unknown) but is learned in phase 2 of the procedure.

\section{NUMERICAL ILLUSTRATIONS}

We have considered Monod and Contois laws (see the graph of the associated functions $v$ on Fig. 1) that provide (for the same values of $D$ and $s_{i n}$ ) the same steady state $s^{e q}=1$. It is then not possible to distinguish between these two functions without changing the value of $D$. Fig. 2

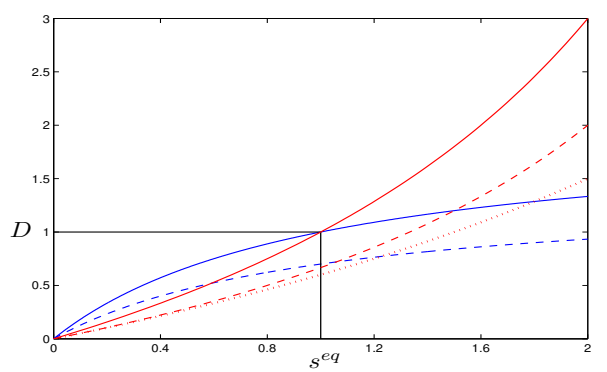

Fig. 1: In blue: Monod's kinetics for $K_{s}=2 ; Y=1 ; s_{\text {in }}=2 ; \mu_{\max }=2$ (plain) and $\mu_{\max }=1.4$ (dashed). In red: Contois's kinetics for $K_{s}=0 ; Y=1$; $k=2 ; s_{i n}=2 ; \mu_{\max }=3$ (plain); $\mu_{\max }=2$ (dashed) and $s_{i n}=3$ (dotted).

illustrates the three phases generated by the procedure (phase 1: blue, phase 2: green, phase 3: red) with the average value $\hat{s}$ (in black). Then Tables I and II (left columns) gives

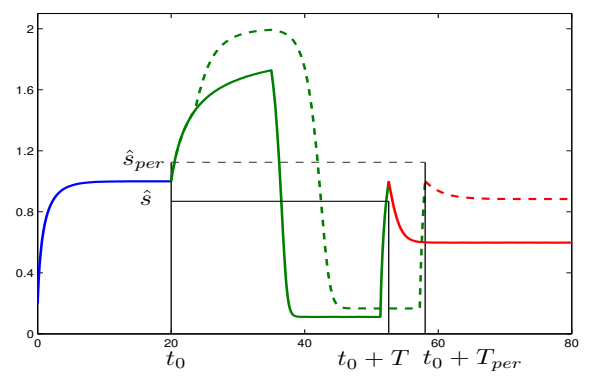

Fig. 2: Solution $s(\cdot)$ generated by the procedure ( for the Monod law) with $s(0)=0.2 ; K_{s}=1 ; \mu_{\max }=2 ; Y=1 ; s_{i n}=2 ; D_{\max }=1.3 ; D_{\min }=0.2$ (in dashed under the perturbation)

the difference $\Delta s:=\hat{s}-s^{\star}$ provided by the procedure, for different values of the durations $\delta_{1}, \delta_{2}$. One can see that a larger period $T$ does not necessarily gives a larger difference $\Delta s$. One has to choose larger values for both $\delta_{1}$ and $\delta_{2}$ to increase the sensitivity of the test.

\begin{tabular}{|c|c|c|c|}
\hline & $\begin{array}{c}\text { Without } \\
\text { perturbation }\end{array}$ & $\begin{array}{l}\text { Perturbation } \\
\text { on } s_{\text {in }}\end{array}$ & $\begin{array}{l}\text { Perturbation } \\
\text { on } \mu_{\max }\end{array}$ \\
\hline & $\begin{array}{c}s_{\text {in }}=2, \\
\mu_{\max }=2\end{array}$ & $\begin{array}{c}s_{i n}=2 \text { and } 3, \\
\mu_{\max }=2\end{array}$ & $\begin{array}{c}s_{\text {in }}=2 \\
\mu_{\max }=2 \text { and } 1.4\end{array}$ \\
\hline$\subseteq \infty$ & $\Delta s=0.24$ & $\Delta s=0.24$ & $\Delta s=0.05$ \\
\hline $\overrightarrow{\|} \|$ & $T=20.4$ & $T=19.2$ & $T=23$ \\
\hline$\sigma^{-12}$ & $\bar{D}=0.81$ & $\bar{D}=0.81$ & $\bar{D}=0.71$ \\
\hline$n \infty$ & $\Delta s=0.26$ & $\Delta s=0.28$ & $\Delta s=0.15$ \\
\hline $\overrightarrow{\|} \|$ & $T=25.6$ & $T=24.2$ & $T=31$ \\
\hline$\sigma^{-12}$ & $\bar{D}=0.90$ & $\bar{D}=0.91$ & $\bar{D}=0.76$ \\
\hline on & $\Delta s=0.23$ & $\Delta s=0.23$ & $\Delta s=0.14$ \\
\hline\|\| & $T=27.4$ & $T=26.2$ & $T=30$ \\
\hline $5 \sigma^{2}$ & $\bar{D}=0.65$ & $\bar{D}=0.65$ & $\bar{D}=0.59$ \\
\hline$n n$ & $\Delta s=0.27$ & $\Delta s=0.29$ & $\Delta s=0.24$ \\
\hline & $T=32.6$ & $T=31.3$ & $T=38$ \\
\hline 505 & $\bar{D}=0.75$ & $\bar{D}=0.76$ & $\bar{D}=0.66$ \\
\hline
\end{tabular}

TABLE I: Results for Monod law: $K_{S}=2 ; Y=1 ; D_{\max }=1.3 ; D_{\min }=0.2$.

\begin{tabular}{|c|c|c|c|}
\hline & $\begin{array}{c}\text { Without } \\
\text { perturbation: }\end{array}$ & $\begin{array}{l}\text { Perturbation } \\
\text { on } s_{i n} \text { : }\end{array}$ & $\begin{array}{l}\text { Perturbation } \\
\text { on } \mu_{\max }:\end{array}$ \\
\hline & $\begin{array}{c}s_{i n}=2, \\
\mu_{\max }=3\end{array}$ & $\begin{array}{c}s_{\text {in }}=2 \text { and } 3, \\
\mu_{\max }=3\end{array}$ & $\begin{array}{c}s_{\text {in }}=2, \\
\mu_{\max }=3 \text { and } 2\end{array}$ \\
\hline$@ \infty$ & $\Delta s=-0.20$ & $\Delta s=-0.55$ & $\Delta s=-0.32$ \\
\hline & $T=19.5$ & $T=19.73$ & $T=22.1$ \\
\hline$\sigma^{-\infty}$ & $\bar{D}=1.47$ & $\bar{D}=1.44$ & $\bar{D}=1.31$ \\
\hline$n \infty$ & $\Delta s=-0.18$ & $\Delta s=-0.45$ & $\Delta s=-0.26$ \\
\hline & $T=24.5$ & $T=24.8$ & $T=29.2$ \\
\hline$\sigma^{-10}$ & $\bar{D}=1.72$ & $\bar{D}=1.69$ & $\bar{D}=1.46$ \\
\hline 으 & $\Delta s=-0.22$ & $\Delta s=-0.51$ & $\Delta s=-0.35$ \\
\hline & $T=26.5$ & $T=26.73$ & $T=29.1$ \\
\hline 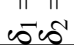 & $\bar{D}=1.11$ & $\bar{D}=1.09$ & $\bar{D}=1.02$ \\
\hline $\ln$ & $\Delta s=-0.23$ & $\Delta s=-0.48$ & $\Delta s=-0.31$ \\
\hline\|\| & $T=31.5$ & $T=31.8$ & $T=36.2$ \\
\hline 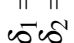 & $\bar{D}=1.36$ & $\bar{D}=1.34$ & $\bar{D}=1.20$ \\
\hline
\end{tabular}

TABLE II: Results for Contois law: $k=2 ; \mathrm{Y}=1 ; D_{\max }=2.7 ; D_{\min }=0.1$.

The interest of the procedure, compared to classical growth identification, relies on its robustness with respect to disturbances. It is well known that continuous cultures are often subject to uncontrolled environmental changes, such as variations of $s_{i n}$, temperature or $\mathrm{pH}$ which directly impact the maximum growth rate $\mu_{\max }$. We have simulated such a sudden change during the phase 2 of the procedure. These changes correspond to the functions $v$ in dashed line on Fig. 1 and modify the $s$-trajectory as depicted in dashed line on Fig. 2. Tables I and II present the results under two kinds of disturbances: change of $s_{i n}$ and change of $\mu_{\max }$. In any case, one can observe that the sign of $\Delta s$ is preserved. Notice that under these changes, the duration $T$ is modified and the $s$ solution between $t_{0}$ and $t_{0}+T$ is no longer periodic, neither for the old or the new functions $v$, but $\Delta s$ is a continuous function of the parameters $s_{i n}, \mu_{\max }$, justifying the robustness of the test.

\section{CONCLUSION}

We have proposed an experiment procedure to test a density dependency in the chemostat, using non-constant dilution rates. This procedure consists in a sequence of a steady state, a periodic operation over only one period, and a 
last steady-state, with the single measurement of the substrate concentration. We have shown its robustness with respect to disturbances on the growth curves during the procedure, demonstrating its interest compared to classical identification methods. For more complex growths for which the function $\mu$ could be neither convex or concave on their whole domain (such as the Hill function [24] or the one from the Microbial Transition State theory [13]), one would need to choose adequately the bounds $D_{\min }, D_{\max }$ in the procedure. This is an open problem that could be the matter of a future work.

\section{APPENDIX}

We recall results from [7] about optimal periodic control. Consider the following dynamical system:

$$
\dot{x}=f(x)+u(t) g(x), u(t) \in[-1,1] \text { and } x(0)=\bar{x},
$$

where $f: I^{\prime} \rightarrow \mathbb{R}, g: I^{\prime} \rightarrow \mathbb{R}_{+}^{*}$ and $I^{\prime}$ is an open interval of $\mathbb{R}$. $f$ and $g$ are assumed to be $C^{1}$ with $f+g>0$ and $f-g<0$ over $I^{\prime}$. Consider now the following optimal control problem:

$$
\min _{u(\cdot)} \frac{1}{T} \int_{0}^{T} \ell(x(t)) d t
$$

where $\ell: I^{\prime} \rightarrow \mathbb{R}$ and $x(\cdot)$ is a $T$-periodic solution of (17) associated with a measurable control $u(\cdot)$ satisfying

$$
\frac{1}{T} \int_{0}^{T} u(t) d t=\bar{u}
$$

where $\bar{u}:=-f(\bar{x}) / g(\bar{x})$. Define $\psi: I^{\prime} \rightarrow \mathbb{R}$ as $\psi:=-\frac{f}{g}$ and $\gamma:=\psi \circ \ell^{-1}$ whenever $\ell^{-1}$ is well-defined, as well as

$$
\tilde{\eta}(x):=\frac{1}{f(x)+g(x)}-\frac{1}{f(x)-g(x)}, x \in I^{\prime} .
$$

It is supposed in [7] that $I^{\prime}$ is invariant by the dynamics (17) with $\psi\left(I^{\prime}\right) \subset[-1,1]$, and that there is a unique $\bar{x} \in I^{\prime}$ s.t.

$$
(\psi(x)-\psi(\bar{x}))(x-\bar{x})>0, x \in I^{\prime}, x \neq \bar{x} .
$$

It is also shown in that when $\ell$ is increasing and $\gamma$ strictly convex increasing, there is a unique $\left(x_{m}, x_{M}\right) \in I^{2}$ s.t.

$$
\int_{x_{m}}^{x_{M}} \tilde{\eta}(x) d x=T, \quad \int_{x_{m}}^{x_{M}} \tilde{\eta}(x) \psi(x) d x=T \bar{u} .
$$

Theorem 3.6 in [7] shows that the bang-bang control whose restriction over $[0, T]$ is defined as

$$
u^{+}(t):=\mid \begin{array}{lll}
+1 & \text { if } & t \in\left[0, \tilde{t}_{1}\right] \\
-1 & \text { if } & t \in\left[\tilde{t}_{1}, \tilde{t}_{2}\right] \\
+1 & \text { if } & t \in\left[\tilde{t}_{2}, T\right]
\end{array}
$$

(where $\tilde{t}_{1}$ is the first time $x$ reaches $x_{M}$ and $\tilde{t}_{2}$ the first time $t>\tilde{t}_{1}$ where $x$ reaches $x_{m}$ ) is optimal (up to a unique time translation defining an optimal control $u^{-}$with same cost).

\section{ACKNOWLEDGMENTS}

The authors thank the Algeria-France program AVERROES for the PhD grant of F.-Z. Tani, the support of the LabEx NUMEV incorporated into the I-Site MUSE and the ANR project THERMOMIC, that gave us the idea to develop this procedure.

\section{REFERENCES}

[1] E. Abulesz and G. Lyberatos, Periodic Optimization of Microbial Growth Processes, Biotech. \& Bioeng., 29, 1059-1067, 1987.

[2] E. Abulesz and G. Lyberatos, Periodic Operation of a Continuous Culture of Baker's Yeast, Biotech. \& Bioeng. 34, 741-749, 1989.

[3] R. Antonelli, J. Harmand and J. P. Steyer and A. Astolfi. Set point regulation of an anaerobic digestion process with bounded output feedback, IEEE Trans. on Control Sys. Tech., 11, 495-504, 2003.

[4] R. Arditi and L. Ginzburg, How species interact? Altering the Standard View on Trophic Ecology Oxord Univerity Press, 1992.

[5] R. Arditi and H. Saiah Empirical Evidence of the Role of Heterogeneity in Ratio-Dependent Consumption, Ecology, 73, 1544-1551, 1992.

[6] G. Bastin and D. Dochain, On-Line Estimation and Adaptive Control of Bioreactors, Elsevier, 1990.

[7] T. Bayen, A. Rapaport and F.-Z. Tani, Optimal periodic control for scalar dynamics under integral constraint on the input, Math. Control. and Related Fields, 2018, to appear.

[8] T. Bayen, A. Rapaport and F.-Z. Tani, Optimal periodic control of the chemostat with Contois growth function, 9th IFAC International Conference on Mathematical Modelling, 21-23 Feb. 2018, IFACPapersOnLine, 51(2), 730-734, 2018.

[9] S. Bittanti, A. Locatelli and S. Rinaldi, Status of periodic optimization of dynamical systems, J. Optim. Theory and Appl., 14, 1-20, 1974.

[10] P. De Leenheer, D. Angeli, and E. Sontag, Crowding effects promote coexistence in the chemostat, J. Math. Anal.pp., 319(1), 48-60, 2006.

[11] D. Contois, Kinetics of Bacterial Growth: Relationship between Population Density and Specific Growth Rate of Continuous Cultures, J. Gen. Microbiology, 21, 40-50, 1959.

[12] A. Corman, G. Carret, A. Pavé, J.P. Flandrois and C. Couix, Bacterial growth measurement using an automated system: Mathematical modelling and analysis of growth kinetics, Ann. Ins. Pasteur / Microbiologie, 137(1), 133-143, 1986.

[13] E. Desmond-Le Qumner1 and T. Bouchez, A thermodynamic theory of microbial growth, ISME J. 8(8), 1747-1751, 2014.

[14] D. Dochain State and parameter estimation in chemical and biochemical processes : a survey. J. of Process Control, 13 (8), 801-818, 2003.

[15] E. Gilbert, Optimal periodic control: A general theory of necessary conditions, SIAM J. Control Optim., 15, 717-746, 1987.

[16] E. Gilbert, Q. Wang, Q. and J.L. Speyer, Necessary and Sufficient Conditions for Local Optimality of a Periodic Process, SIAM J. Control Optim., 28, 482-497, 1990.

[17] J. Harmand and J.-J. Godon, Density-dependent kinetics models for a simple description of complex phenomena in macroscopic massbalance modeling of bioreactors Ecol. Model., 200, 393-402, 2007.

[18] J. Harmand, C. Lobry, A. Rapaport and T. Sari, The Chemostat: mathematical theory of microorganisms cultures, ISTE Wiley, 2017.

[19] P. Hoskisson and G. Hobbs, Continuous culture - making a comeback Microbiology, 151, 3153-3159, 2005.

[20] E. Krichen, J. Harmand, M. Torrijos, J.-J. Godon, N. Bernet and A. Rapaport, High biomass density promotes density-dependent microbial growth rate, Biochemical Engineering Journal, 130, 66-75, 2018.

[21] C. Lobry, F. Mazenc and A. Rapaport, Persistence in ecological models of competition for a single resource, Comptes-rendus Mathématiques, Académie des Sciences, Paris, 340(3), 199-204, 2005.

[22] C. Lobry, F. Mazenc and A. Rapaport, Persistence in Ratio-Dependent Models of Consumer-Resource Dynamics, Electronic Journal of Differential Equations, 15, 211-220, 2005.

[23] J. Monod, La technique de culture continue: Théorie et applications, Ann. Inst. Pasteur, Lille, 79, 390-410, 1950.

[24] H. Moser, The Dynamics of Bacterial Populations Maintained in the Chemostat, Carnegie Institution of Washington Publication, 1958.

[25] A. Novick and L. Szilard, Experiments with the chemostat on spontaneous mutations of bacteria, Nat. Acad. Sci., 6(12), 708-719, 1950.

[26] A. Rapaport and J. Harmand. Robust regulation of a class of partially observed nonlinear continuous bioreactors, J. Process Control, 12, 291-302 2002.

[27] J. Sieber, A. Rapaport, S. Rodrigues and M. Desroches. A new method for the reconstruction of unknown non-monotonic growth function in the chemostat, Bioprocess and Biosystems Eng., 36, 1497-1507, 2013.

[28] H. Smith and P. Waltman, The Theory of Chemostat - Dynamics of microbial competition, Cambridge University Press, 1995.

[29] N. Ziv, N. Brandt and D. Gresham, The use of chemostats in microbial systems biology, J. Vis. Exp, 80, 1-10, 2013. 